\title{
Very Tight Coupling of Wireless LANs and UMTS Networks: A Technical Challenge and an Opportunity for Mobile Operators
}

\author{
Manfred Litzenburger, Hajo Bakker, Stephen Kaminski, Klaus Keil \\ Alcatel Research and Innovations, D-70430 Stuttgart, Germany
}

Abstract: Coupling of Wireless LANs like IEEE 802.11a/b or HIPERLAN/2 systems with Public Land Mobile Networks (PLMNs) like GSM or UMTS offers benefits for both operators and users. Coverage and capacity of an operator's network can be extended with equipment expected to be considerably cheaper than for PLMN coverage. Thus, the operator can participate in the expected WLAN boom. Its customer experiences homogenous access to services with high data rate. PLMNs and WLANs should be considered as complementary systems: PLMNs provide universal coverage and high mobility support, while pico-cellular WLANs will be applied in hot spot areas, offering high data rates (up to about $45 \mathrm{Mbit} / \mathrm{s} / \mathrm{cell}$ in HIPERLAN/2). Several levels of interworking are currently being defined by the relevant standardisation bodies. Tight and Very Tight Coupling integrate the WLAN into a unified Radio Access Network. But PLMNs and WLANs are based on different design philosophies. Chances, problems, and possible solutions are discussed in this paper.

Key words: Wireless LAN, Tight Coupling, Very Tight Coupling, IEEE802.11, HIPERLAN/2, Multistandard Radio Access, Radio Resource Management

\section{INTRODUCTION}

Coupling of WLANs and PLMN systems can be implemented in different ways. 3GPP defines six stages of coupling with an increasing level of interworking [1]. The scenarios range from Open Coupling (level 1, basically constituting two separate access systems with common billing only) over Loose Coupling (level 2 and 3, additionally common Authentication, Authorisation, and Accounting (AAA) services) to Tight

The original version of this chapter was revised: The copyright line was incorrect. This has been corrected. The Erratum to this chapter is available at DOI: 10.1007/978-0-387-35618-1_37 
(level 4 and 5) and Very Tight Coupling (level 5 and 6). Several field trials currently are implementing and testing the Open and Loose approach (e.g., [2]). In this paper we consider coupling with UMTS. With Tight Coupling the WLAN Access Point (AP) is connected like a Radio Network Controller (RNC) to the Serving GPRS Support Node (SGSN) in the Core Network (CN), while with Very Tight Coupling it is connected to the RNC and, thus, gets an integral part of the UMTS Radio Access Network (RAN). An Interworking Unit might be added, which will be explained later. The complexity of implementation, of course, increases with the level of interworking. Tight or Very Tight Coupling means a single account for the subscriber and at least service continuity when roaming between the two access technologies. This requires, of course, the use of dual mode user terminals. The basic architectures of WLAN-UMTS Tight and Very Tight Coupling are shown in Fig. 1. We focus here on Very Tight Coupling. It provides the possibility to offer a homogenous service to the subscriber and to perform inter-system Radio Resource Management (RRM).Very Tight Coupling is an important step towards a Multistandard RAN (also called heterogeneous or co-operative networks) that integrates separate air interfaces into a single common network [3].

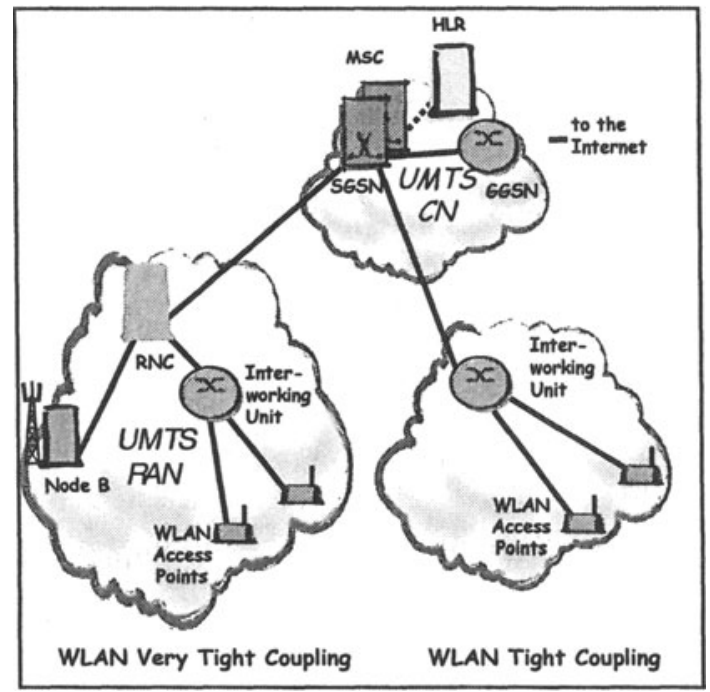

Figure1. Architecture of Tight and Very Tight WLAN coupling to UMTS

An important issue is the ownership of the WLAN. While with Open and Loose Coupling the two access networks may be owned by separate operators, the most probable solution for Very Tight Coupling is that the UMTS operator owns also the WLAN part and, thus, keeps its customers within its own network. 


\section{DESIGN CRITERIA}

Important criteria when designing a Very Tight Coupling architecture are:

- Use of off-the-shelf WLAN APs and Wireless Terminals (WTs), or, at least, no hardware modifications should be necessary. This enables the user to access WLAN systems, independently of the coupling scenario, just by loading individual software drivers.

- As few as possible changes in the UMTS infrastructure to offer the UMTS operator a cost-efficient coupling solution.

- Independence from the actual WLAN standard, i.e., the requirements for the Very Tight Coupling architecture should be defined by UMTS standardisation bodies (i.e..3GPP) while extensions of the WLAN standard should be specified individually by the different WLAN standardisation bodies.

- A common Radio Resource Management to enable a better utilisation of the UMTS operator's radio access network by load distribution between UMTS and WLAN.

- Transparency towards the end user with respect to services which are billed independently of the used radio access system, otherwise a manual selection has to be offered.

- Seamless service continuity with respect to data loss and break time during the switch between the different radio access technologies.

WLANs can be applied for coverage extension of UMTS, especially where ubiquitous UMTS coverage is not available, e.g., within buildings. All necessary functions for connection set-up, paging, etc. will be provided via the WLAN air interface as well. Thus, all UMTS transport channels and their mapping to MAC-d or MAC-c/sh, resp., have to be provided and transported by the WLAN systems. When coupling a WLAN to a UMTS infrastructure, two different philosophies collide. WLANs pursue a plugand-play approach for fast and flexible reconfigurability. This enables the operator to cope with changes in configuration and topology, which may be required quite often (e.g., at fairs, exhibitions, offices). The reconfigurability is supported by the Dynamic Frequency Selection (DFS) [5] which at least is mandatory for WLANs operated in Europe. The DFS algorithm assures that each WLAN AP selects out of the range of available frequencies the best suited one with respect to interference. The frequency selection made by each WLAN AP is based on filtered interference measurements performed by the WLAN AP and its associated WTs. The DFS is carried out during start-up of the WLAN network, removal or addition of WLAN APs or the occurrence of external interferers. UMTS networks, on the other hand, are 
assumed to have a rather static topology. The cell- and frequency-planning is performed offline and every change requires Operation and Maintenance (O\&M) activities for reconfiguration. Thus, a UMTS network operator will not accept that each modification within a WLAN network is visible inside the RNC, i.e., some filtering function hiding the internal structure of the WLAN is required.

In general a WLAN AP is rather intelligent and autonomous compared to a UMTS Node B, because in the UMTS RAN the intelligence is concentrated in the RNC. E.g., if we consider mobility: In a WLAN the handover is initiated by the terminal, which selects a new AP. The AP has its own RRM and connection admission control functionality. In UMTS the RNC initiates and controls handovers based on link quality measurements provided by the terminal and the Node B. In order to realise a common RRM it is necessary either to modify the WLAN handover procedures, which might be difficult with respect to the different WLAN standardisation bodies, or to 'hide' the mobility inside the WLAN network towards the RNC in such a way that the RNC still can control the traffic in the radio cells. Another important issue is the transport of user data traffic. Compared to UMTS access networks, WLANs provide a much larger bandwidth to the subscriber. This traffic will have to be transferred through the network the WLAN is connected to. A higher degree of interworking involves a larger number of UMTS network elements that have to provide the necessary capacity with respect to processing power and interface capabilities, which can be currently considered as to be up to around $30 \mathrm{Mbit} / \mathrm{s}$ per connected WLAN AP for the advanced WLAN standards. Emphasis should be taken to concentrate this impact only to the user plane, e.g. by separation of the user and control plane in the RNC.

\section{IMPLEMENTATION APPROACHES}

There are several possibilities for the realisation of the Very Tight Coupling. Generally, an RNC controls a large area in a UMTS network which may contain several hot spots to be covered with WLANs, possibly located far away from the RNC location. Due to the pico-cellular nature of WLANs this means a huge number of WLAN APs has to be connected to and controlled by one RNC. Therefore it makes sense to introduce an Interworking Unit (IWU) between the RNC and the WLAN APs. This IWU, located at the WLAN hot spot, can either act as a pure traffic concentrator, i.e., without own intelligence, or additionally an IWU control plane is introduced to enable a control and supervision functionality of the WLAN APs. However this approach does not correspond with the UTRAN network topology, as 3GPP does not define any further active network element (with 
respect to processing and conversion of protocols) between the RNC and an UMTS Node B. Therefore, we are proposing that the IWU represents towards the RNC some kind of Node B serving one or more 'WLAN-UMTS radio cells' which correspond to the WLAN hot spot area. In this case the WLAN APs might be seen as remote installed layer 1 modules of a UMTS Node-B. For large hot spot areas the IWU should act as a Node B with multiple sectors consisting of multiple 'WLAN-UMTS radio cells'. This solution is applicable in the case of grouping several APs within a floor of a building or any other similar configuration. Each 'WLAN-UMTS radio cell' is controlled individually by the RNC. Thus a better supervision of the WLAN traffic in specific areas is achieved. The interface between the RNC and the IWU is very similar to the UMTS Iub interface between RNC and Node B and should be based on available technology, e.g., ATM. The IWU functionality might be included in one specific WLAN AP or the IWU represents a standalone device. As the distance between the IWU and the WLAN APs is limited, Ethernet connections could be applied.

Compared to WLAN APs which are directly connected to an RNC, the approach based on an IWU offers the following benefits:

- The kind of WLAN network (HIPERLAN/2 or IEEE $802.11 \mathrm{a} / \mathrm{b}[6,7]$ ) is hidden towards the RNC, i.e., there is a defined split between the UMTS responsibility and the WLAN responsibility with respect to standardisation. The interface between the RNC and the IWU has to be specified by 3GPP, while the interface to the APs has to be specified by the WLAN standardisation bodies, based on interworking requirements which have to be defined by 3GPP. This means that the IWU controls common interworking procedures within the WLAN network, the implementation of theses procedures might differ between the individual WLAN systems. Other UMTS functions, e.g., authentication, security etc., which are terminal related and which are required for the very tight interworking have of course to be defined by 3GPP. As these latter functions are located within higher protocol layers, they are independent of the underlying WLAN technology. These functions should be transparent to the IWU to reduce the complexity of the IWU as much as possible.

- The WLAN topology and any configuration changes (removal or addition of APs) inside the WLAN network is hidden towards the RNC.

- The WLAN mobility is hidden towards the RNC, as all mobile WLAN terminals within the area of an IWU are still in one or more 'WLANUMTS radio cells'. Thus, there is no need to modify the handover algorithm of the WLAN systems.

- The Very Tight Coupling can be realised without hardware modifications of the WLAN terminals and the WLAN APs. 
WLAN Very Tight Coupling can be considered as a step towards a common RRM. The RNC has to control resources in the WLAN in order to support and control mobility and connection admission. Forced handovers from UMTS to WLAN and from WLAN to UMTS should also be possible to perform load balancing. Thus, for a common RRM, the IWU has to provide continuously a load report to the RNC which comprises the current resource utilisation of the complete 'WLAN-UMTS cell'. This load report varies as it is based on internal WLAN functionality like, e.g., change of the modulation scheme, handover of WLAN terminals between 'WLAN-UMTS radio cells' (which is not controlled by the RNC), or 'on the fly' installation or removal of WLAN APs based on the plug and play principle.

To enable a seamless handover in the case of a UMTS-to-WLAN or WLAN-to-UMTS transition all WLAN data and control information should be mapped to UMTS logical channels. The protocol stacks defined in this paper are based on the approach to use the IP layer provided by the WLAN network to transport UMTS MAC-d and MAC-c/sh PDUs. Based on this assumption, each WLAN system offering the possibility to transport IP traffic can be used for this Very Tight Coupling approach.

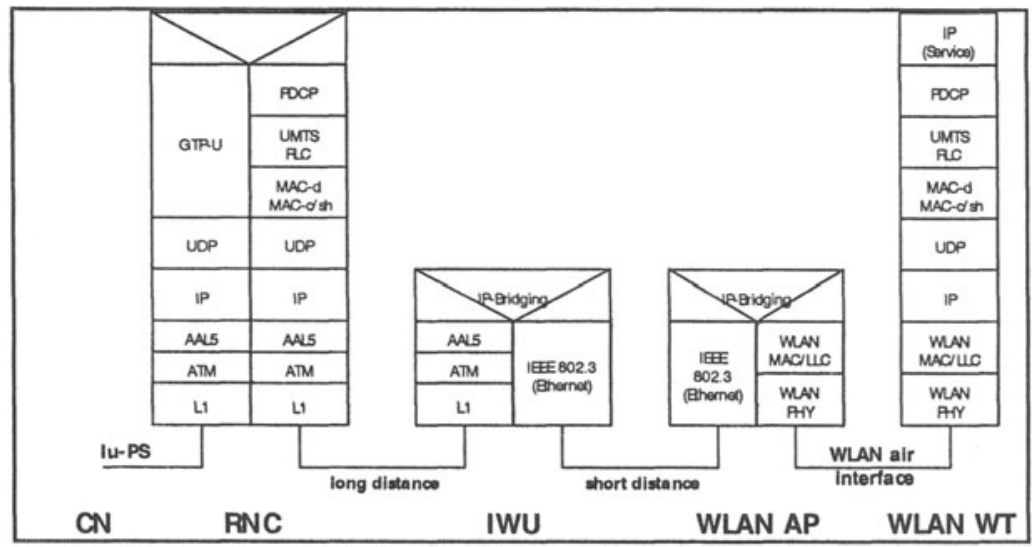

Figure2 User plane protocol stack for Very Tight Coupling

A protocol stack for the user plane depicted in Fig.2 for the case of a WLAN-only WT. For details of the individual UMTS protocol entities we refer to [4]. Above the UDP layer the normal UMTS stack is applied, i.e., the architecture is completely transparent, the IP-tunnelling mechanism (PDCP, GTP-U) between the terminal and the CN is not affected. The WLAN is just used as another air interface. For a dual mode terminal equipped with a WLAN and a UMTS interface a second protocol stack below the MAC-d layer should be drawn, but as there is no difference to the standard UMTS stack, it is omitted in Fig.2. The frame protocol (FP) between the RNC and 
the Node B, which in UMTS is necessary for the multiplexing of the MAC PDU is removed. Instead the individual IP connections between the RNC and the IWU are used for this functionality. The corresponding control plane for the "intelligent IWU" approach is depicted in Fig.3.

The generation and the analysis of the load report between the IWU and RNC is processed within the 'IWU Control' layer. The NWAP protocol (Node-W Application Part, an extension of the UMTS NBAP protocol) might be used as transport function (see Fig.3). The load report represents the total load of the overall 'WLAN-UMTS radio cell', but there is still the possibility that the traffic inside a 'WLAN-UMTS radio cell' is not distributed homogeneously, e.g., a huge number of WLAN WTs communicate via one AP while other APs serve only a few WTs. In this case the IWU has to distribute the load by means of a forced handover within the 'WLAN-UMTS radio cell'. This function is included in the WLAN RRC layer. The IWU also supports the handling of the complementary handover strategies of UMTS and WLAN systems: Within UMTS the handover is controlled by the RNC for all CS and PS services transported in the DCHs. In contrast, the handover decision algorithm in WLAN terminals is completely independent from the WLAN AP, i.e. the AP is not involved, a WLAN terminal contacts without any control of the WLAN network the selected new AP. We propose the following concept:

- The RNC shall not have any control with respect to handover of inside a "WLAN-UMTS radio cell", i.e. horizontal handover.

- If a WLAN terminal moves from one "UMTS-WLAN radio cell" to another controlled by the same or another IWU the RNC is informed.

- Each vertical handover is controlled by the RNC, i.e., the RNC informs the IWU that a dual mode terminal will leave or enter the 'UMTSWLAN radio cell'.

\section{BENEFITS FOR OPERATORS}

The Very Tight Coupling approach integrates WLANs together with UMTS into one single access network, owned and operated by one operator. It enables common optimisation of bandwidth resources within the network as well as on the air. It is to be expected that WLANs will proliferate in the coming years and take a considerable share of the wireless data traffic. With Very Tight Coupling the operator can build upon his established customer base and keep its customers within its own network to offer broadband services complementary to UMTS. 


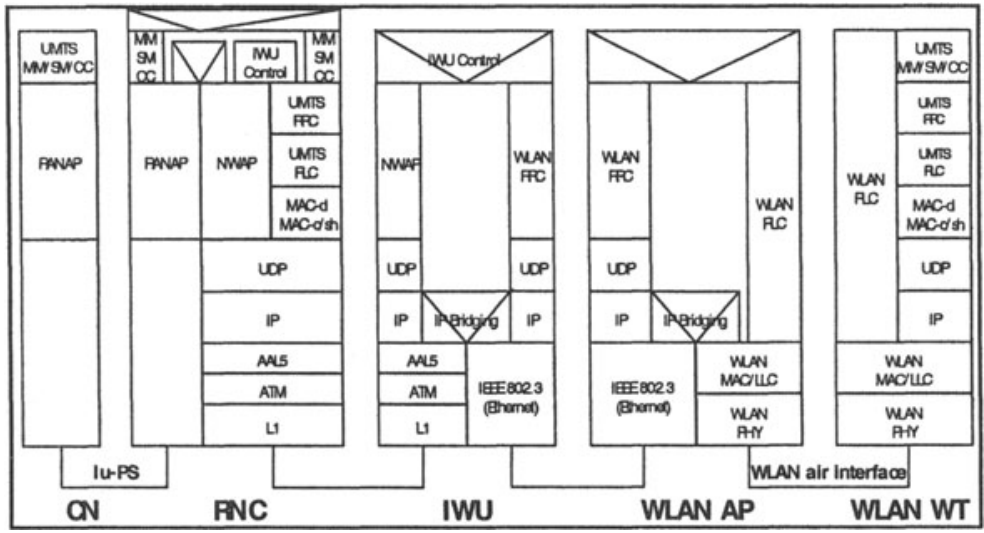

Figure 3 Control plane protocol stack for Very Tight Coupling

\section{SUMMARY}

Tight and Very Tight Coupling are the most interesting options for PLMN operators for integrating WLANs into their networks and extending the services offered to their customers. However, a lot of issues remain to be solved, so it is not to be expected before UMTS Release 6, whereas Open and Loose Coupling can be realised with today's equipment. With Very Tight Coupling UMTS and WLAN air interfaces are integrated into one single RAN, allowing a real common Multistandard Radio Resource Management for overall optimisation of the usage of radio as well as components resources. The end user experiences an universal and homogenous access to services without having to care about different technologies, accounts, or procedures.

\section{REFERENCES}

[1] 3GPP (TSG SA) TS 22.934 V.1.0.0: "Feasibility study on 3GPP system to Wireless Local Area (WLAN) interworking", March 2002.

[2] J. Ala-Laurila, J. Mikkonen, J. Rinnemaa: "Wireless LAN Access Network Architectures for Mobile Operators," IEEE Commun. Mag., Nov. 2001, pp. 82 - 89.

[3] Wireless World Research Forum (WWRF): "The Book of Visions 2001", http://www.wireless-world-research.org, Dec. 2001.

[4] 3GPP (TSG RAN), TS 25.401 V3.8.0: "UTRAN Overall Description (Release 99)", Sept. 2001.

[5] ETSI; HIPERLAN Type 2; Data Link Control (DLC) Layer; Part 2: Radio Link Control (RLC) sublayer; TS 101 761-2 V1.3.1 (2002-01)

[6] http://www.etsi.org/frameset/home.htm?/technicalactiv/Hiperlan/hiperlan2.htm

[7] http://standards.ieee.org/wireless/overview.html\#802.11 